\title{
Interactive Aggregation/Disaggregation Dichotomic Sorting Procedure for Group Decision Analysis Based on the Threshold Model
}

\author{
Andrej BREGAR, József GYÖRKÖS, Matjaž B. JURIČ \\ University of Maribor, Faculty of Electrical Engineering and Computer Science \\ Smetanova 17, SI-2000 Maribor, Slovenia \\ e-mail: andrej.bregar@uni-mb.si,jozsef.gyorkos@uni-mb.si,matjaz.juric@uni-mb.si
}

Received: March 2007

\begin{abstract}
In this paper, a new multi-criteria decision-making procedure is presented, which captures preferential information in the form of the threshold model. It is based on the ELECTRE-like sorting analysis restricted by the localization principle, which enables high adaptability of the decision model and reduces the cognitive load imposed on the decision-makers. It lays the foundation for the introduction of three concepts that have been previously insufficiently supported by outranking methods - semiautomatic derivation of criteria weights according to the selective effects of discordance and veto thresholds, convergent group consensus seeking, and autonomous multiagent negotiation. The interdependent principles are justified, and the methodological solutions underlying their implementation are provided.
\end{abstract}

Keywords: decision-making, group decisions and negotiations, pseudo-criterion, outranking relation, preference aggregation/disaggregation, nonlinear optimization, fuzzy sets, agents.

\section{Introduction}

Multi-criteria decision analysis is a widely used methodology in science, engineering and management (Triantaphyllou and Baig, 2005). It refers to making preference decisions over the available alternatives that are characterized by multiple, usually conflicting criteria. Often, the decision-makers have to deal with insufficiently accurate and uncertain data (Huynh et al., 2006; Peldschus and Zavadskas, 2005), or have to engage into group negotiations in order to reach a consensus or a compromise solution (Raiffa et al., 2002).

There exist several approaches to multi-criteria decision analysis. Among the most popular are the utility theory (Keeney and Raiffa, 1976), the Analytic Hierarchy Process (Saaty, 1980; Triantaphyllou, 2000), the ideal solution based methods, such as TOPSIS (Zavadskas and Zakarevicius, 2006), qualitative or ordinal scale evaluations (Larichev, 2001; Moshkovich et al., 2002; Petrovsky, 2001), and methods that capture preferential information in the form of threshold model (Roy, 1996). It has been proven that the concept of pseudo-criterion, which is founded on the indifference and preference thresholds, deals in an effective and practical way with imprecision, indetermination and uncertainty 
of available numerical data (Miettinen and Salminen, 1999). The treatment of pseudocriteria results in the construction of outranking relations on pairs of alternatives, or between alternatives and reference profiles that delimit predefined categories/classes. In this way, four distinct situations - strong/weak preference, indifference and incomparability are modelled obeying the axiom of limited comparability. Some evidence that it is unrealistic to assume comparability of options as a general case for a rational decision-maker has been provided by Rauschmayer (2001).

The principal outranking methods belong to the PROMETHEE (Brans and Vincke, 1985 ) and ELECTRE (Roy, 1991) families. Several extensions and applications of these methods have been presented in the literature (Azar et al., 2001; Theil and Mroz, 2001). Yet, classical decision-making procedures, which elicit preferential information according to the nature of pseudo-criteria, have a disadvantage that they demand from the decision-makers to set a lot of parameters, putting a substantial cognitive load on them. Moreover, two additional drawbacks appear as a consequence of many required inputs: the quantitative model is insufficiently adaptable, and an inadequate insight into the derivation of results is given. To overcome these difficulties, several interactive methods have been introduced.

The aggregation/disaggregation approach (Jacquet-Lagrèze and Siskos, 2001) has been applied to the ELECTRE TRI method (Mousseau et al., 2000) using outranking relations with the purpose of sorting. A mathematical programming problem is solved to infer preferential parameters from a set of assignment examples based on holistic judgments provided by the decision-maker (Mousseau and Slowinski, 1998). Because the simultaneous derivation of all parameter values requires solving a non-linear program with non-convex constraints, only a subset of parameters is inferred at a time, while maintaining remaining ones fixed (Ngo The and Mousseau, 2002). Dias et al. (2002) argue that inference programs should be considered as problems to be solved several times in an interactive learning process through which the decision-maker continuously revises his preferences as he obtains the results from the model. Their method integrates parameter inference with robustness and consistency analysis.

Miettinen and Salminen (1999) have proposed an ELECTRE III based search procedure in the criteria weight space. It finds the weight vector according to which a chosen alternative is ranked as the best one. Jaszkiewicz and Slowinski (1997) have considered choice problems with large alternative sets by describing three interactive exploration procedures. Interactive trichotomy segmentation (Jaszkiewicz and Ferhat, 1999) is founded on the localization principle. Preferences are modelled in the neighbourhood of a single reference profile in the nondominated set. The model accepts good alternatives, rejects uninteresting ones and defines alternatives that can neither be accepted nor rejected with regard to the available information.

The method, which is introduced in this paper, uses the localization principle in order to reduce the cognitive load imposed on the decision-makers, and to enable high adaptability of the decision model. Yet, contrary to the aforementioned pseudo-criterion based approaches, it does not focus solely on providing interaction, iterativeness and analytical capabilities, but strives to attain two further objectives: 
- group consensus seeking that relies on the aggregation/disaggregation approach and can be easily applied to fully automated agent based negotiation, and

- semiautomatic derivation of criteria weights according to the selective strengths of veto and discordance thresholds.

The rest of the paper is organized as follows. In Section 2, the reasons for the introduction of three proposed principles - problem localization, weight derivation, and group consensus seeking - are discussed. The methodological solutions underlying the implementation of these interdependent concepts are explained in Sections 3 through 6, respectively. Finally, Section 7 concludes the paper by giving a resume and some directions for further work.

\section{Justification of the Proposed Principles}

\subsection{Dichotomic Sorting Based Problem Localization}

According to Jaszkiewicz and Ferhat (1999), specifying a single reference profile, which is a vector of desired values on criteria domains, and searching for alternatives better than this profile is one of the most natural ways to solve multi-criteria decision-making problems. This approach is considered as sorting, since it refers to the absolute assignment of a set of options into existing ordinal categories/classes (Zopounidis and Doumpos, 2002). While $m \cdot(m-1) / 2$ pairwise comparisons between alternatives have to be made in the case of ranking analysis, sorting has the advantage that only $m$ pieces of information about class memberships suffice. As the global problem of assigning alternatives to $p+1$ ordered categories is further reduced to the dichotomic partition of the solution set, several additional benefits appear:

- Providing a single referential value on each criterion domain and a single value of each threshold is cognitively much easier for the decision-maker than setting $p$-times more fixed inputs or defining functional thresholds, which are used by a global preference model.

- Because of mental and time constraints, the decision-maker is rarely capable of altering many reference vectors at once. It is therefore difficult for him to figure out how different profiles affect alternative evaluation. But when he concentrates on only one profile instead, it is relatively easy for him to modify referential values. By doing so, he can tighten or loosen demands and see what effect this has on selection. As a consequence, learning about the problem situation, the model, and advantages/weaknesses of alternatives is improved.

- As the dispersion of alternatives across classes is reduced, comparability of individuals' results is increased.

- The unification of opinions becomes an easy task, because fewer parameters are required. 


\subsection{Group Consensus Seeking}

There exist many methods for group decision-making (Matsatsinis and Samaras, 2001), yet only a few capture preferences in the threshold form. Leyva-Lopez and FernandezGonzalez (2003) give some criticism of the existing approaches. They argue that these techniques rest on a poor heuristic, which makes a decision about the consensus solution difficult to support. The PROMETHEE method (Brans et al., 1997) cannot guarantee unanimity among group members because it uses a net flow weighted sum function and has a compensatory nature. It is, however, supplemented by the GAIA analysis, which can help in reaching a compromise. The idea of GAIA has been further developed by Espinasse et al. (1997). Their approach relies heavily on the guidance of a human moderator who has to interpret six types of GAIA planes. Although a set of interpretation rules is provided, this task is difficult and complex. Colson (2000) has introduced JUDGES, a system which compares rankings of team members. It uses four visual aids to expose disagreements. Jabeur et al. (2004) have defined a procedure to obtain a collective preorder from partial preorders of decision-makers, who can apply either the ELECTRE III or the PROMETHEE method. A version of ELECTRE TRI for groups exists as well (Dias and Clímaco, 2000). It focuses on finding the best and the worst class that an alternative may attain with regard to constraints on individually set imprecise parameters. Finally, several variations of Kendall's $\tau$ ensure a compromise by determining the correlation between rank-orders (Emond and Mason, 2002).

Most of these approaches have a disadvantage that a very complex discussion during group meetings is required. The problem solving process thus has to be very well structured, and a responsible, potentially overly demanding role has to be imposed on the human moderator, especially when the group is large or when there exist many contradicting scenarios. This weakness is additionally emphasized by the fact that the decision support system is incapable of advising participants on how to adjust inputs when considerable discrepancies between personal opinions emerge. Considering the abovementioned drawbacks, an active mechanism for iterative group consensus seeking is needed that would take into account the following demands: all preferential parameters are important in group decision-making, the level of consensus has to be known, the decision-making process has to be democratic, the activity of the human moderator should be reduced to the minimum, and the system should be able to tell the decision-maker how he can modify his input parameters so that they will correspond to the preferences of the whole group. It is crucial for a convergent negotiation procedure to adopt the aggregation/disaggregation paradigm (Bregar, 2005). According to evaluated alternatives that reflect the global preference structure of both the individual decision-maker as well as the complete group, it has to automatically adjust the parameters of the model so that it becomes capable of reproducing the agreed upon consensual solutions.

Recently, a powerful methodology that combines the principles of group decisionmaking, sorting, outranking and aggregation/disaggregation has been introduced (Demart et al., 2006). It has been implemented with the IRIS system (Dias and Mousseau, 2003). It is efficient, but has a few limitations which are addressed by the interactive procedure proposed in this paper: 
- it does not concentrate on other preference parameters than criteria weights;

- the determination of criteria weights is based on holistic assessments of alternatives, while the values of other preference parameters, such as veto thresholds, are not considered;

- a complicated and time consuming discussion of group members is required, which relies heavily on the human mediator and has to be structured as a Delphi process (Turoff and Hiltz, 1996) for which no outline has been provided;

- the mechanism aims primarily at directing the decision-makers, and hence does not enable fully automated agent based negotiation;

- the mechanism does not identify the decision-maker who has to conform to the other group members, leaving this judgement to the moderator;

- the robustness of the decision is not measured with regard to the allowed deviation/change of preferential parameters.

Majority of aggregation/disaggregation techniques for group decision-making are based on the multi-attribute utility theory. They apply the UTA/UTASTAR methods (Jacquet-Lagrèze and Siskos, 2001) to derive partial value functions of individual decision-makers. Afterwards, they aggregate utilities of alternatives with some averaging operator. Matsatsinis et al. (2005) have found that such representations of group preferences guarantee neither a consensus nor a good compromise because individual assessments may be considerably different. Therefore, they have incorporated several criteria to measure the decision-makers' satisfaction over the aggregated rank-order of alternatives. Since they have consequently increased the cognitive load, it is a necessity for any efficient new method not to average the individuals' evaluations.

\subsection{Semiautomatic Weight Derivation}

In the past, guidelines for specifying realistic values of the indifference, preference and veto thresholds have been introduced (Rogers and Bruen, 1998a). On the other hand, the assessment of criteria importance weights remains a hard and time consuming task. Because it is difficult to elicit weights, a couple of structured techniques have been defined, such as the Analytic Hierarchy Process (Saaty, 1980), Simos' procedure (Figueira and Roy, 2002) or the "resistance to change" grid (Rogers and Bruen, 1998b), that aim at reducing the cognitive load, and that help people make reasonable estimates. Most existing methods, however, have their origin in the multi-attribute utility theory. The direct rating method (von Winterfeldt and Edwards, 1986) requires the decision-maker to provide exact weights by considering relative preferences for swings from the worst to the best options on each attribute, or by comparing attributes to the least significant one. This method has been advanced by several approaches that allow for the specification of linear constraints on weight intervals in order to deal with imprecise judgments and incomplete information (Eum et al., 2001; Mateos et al., 2003). By applying the trade-off procedure (Keeney and Raiffa, 1976), the decision-maker determines probabilities for which he is indifferent between gambles and sure consequences. Salo and Hämäläinen (2001) have proposed the PRIME method combining the trade-off approach with AHP-like decomposed ratio judgments. Several techniques that translate criteria importance ranks into 
weight approximations have also been introduced (Roberts and Goodwin, 2002). In addition, optimization programs that derive weights on the ground of evaluated alternatives have been defined (Miettinen and Salminen, 1999).

These approaches are, however, incapable of automatic weight determination according to a given problem situation, which is reflected through values of other input parameters. The presented research bridges this gap. A mechanism is proposed that obtains criteria weights by considering the selective influence of veto and discordance thresholds.

Although a relationship between the veto threshold $v_{j}$ and the criterion weight $w_{j}$ has been discussed in the past (Rogers and Bruen, 1998a), it has neither been mathematically founded nor practically applied. The $v_{j}$ threshold characterises the conditions under which a criterion can prevent an outranking relation. It conveys the idea that outranking of $b$ by $a$ is vetoed when $a$ performs much worse than $b$ for any criterion. If the difference in value between $a$ and $b$ exceeds the preference threshold $p_{j}$, or the discordance threshold $u_{j}$ (Mousseau and Dias, 2003), respectively, the discordance rises above zero. Similarly, the magnitude of criterion valuation difference at which the outranking relation is with certainty opposed is represented by the $v_{j}$ threshold. This has the effect of neutralising the mechanism of veto for the criteria of lesser importance while making it an essential factor for the most significant ones. The nearer $v_{j}$ is to $p_{j}$, the lower the criterion valuation difference is at which the veto is imposed, and the more the $v_{j}$ threshold affects the overall outranking of one option over another. In this way, the correlation between decreasing values of $v_{j}-p_{j}$ and increasing values of $w_{j}$ is implied, and it can be argued that the veto threshold is connected to the importance rating of a criterion.

In the case when available alternatives are sorted into $p+1>2$ categories, the intercriterion veto influence is locally limited to two adjacent categories. This means that various settings of the veto threshold cause the reassignment of an alternative to at most the adjoining category. Results of the alternative sorting analysis are therefore largely dependent on referential values of $p$ profiles, and not so much on the $v_{j}$ thresholds' magnitudes. For this reason, the introduced weight derivation mechanism is restricted by the localization principle.

The proposed procedure to specify the criteria weights relies upon the assumption that the importance of a criterion is closely related to its ability to exclude cases from the category of good alternatives. The discordance test is thus employed as the basis for the weight derivation procedure. It is essential, though, that the complementary concordance test should not have any influence on the importance of a criterion. This test is founded on the indifference and preference thresholds, which provide intracriterion preferential information. Their effect has to be compensated in order to deal with imprecision, indetermination and uncertainty of data. Because, by definition, the weights are introduced specifically to determine the compensation rates, it is their task to restrain the effect of the indifference and preference thresholds, and not the opposite.

\section{Dichotomic Alternative Sorting}

In order to implement the localized alternative sorting analysis, a slightly modified version of the ELECTRE TRI method is used. The set of alternatives is partitioned into two 
exclusive categories $-C^{+}$and $C^{-}$. All acceptable choices belong to the positive category $C^{+}$, while unsatisfactory ones are members of the negative category $C^{-}$. The categories are delimited by the profile $b$, which is defined as a vector of $n$ referential values on criteria domains. Let this vector be denoted as $\left(g_{1}(b), g_{2}(b), \ldots, g_{n}(b)\right)$. Let similarly $g_{j}\left(a_{i}\right)$ denote the value of an alternative $a_{i} \in A$ that is measured with regard to a criterion $x_{j} \in X$. The assignment of each alternative to either the positive or the negative class then results from comparisons of values $g_{j}\left(a_{i}\right)$ with values $g_{j}(b)$, where $j=1, \ldots, n$. Because numerical evaluations are subject to imprecision, indetermination and uncertainty, and because people are unable to perceive small differences in data, it is essential that an alternative does not have to outperform the profile on all criteria to be sorted into the positive class $C^{+}$. Weaknesses on some criteria are therefore admissible and can be compensated with advantages on other criteria. Two parameters allow for compensation the indifference threshold $q_{j}$ and the preference threshold $p_{j}$. On the basis of these thresholds, two valued outranking relations are built for each criterion expressing the degree to which the alternative $a_{i}$ outperforms the profile $b$ ( $b$ outperforms $a_{i}$, respectively):

$$
\begin{aligned}
& s_{j}\left(a_{i}, b\right)=\max \left(\min \left(\left(g_{j}\left(a_{i}\right)-g_{j}(b)-q_{j}\right) /\left(p_{j}-q_{j}\right), 1\right), 0\right), \\
& s_{j}\left(b, a_{i}\right)=\max \left(\min \left(\left(g_{j}(b)-g_{j}\left(a_{i}\right)-q_{j}\right) /\left(p_{j}-q_{j}\right), 1\right), 0\right) .
\end{aligned}
$$

In real-life problems, alternatives having poor values are not taken into consideration. This means that certain criterion weaknesses are not accepted to be compensated by good values on some other criteria. To model partial incompensation, the discordance concept is applied. It is based on the veto threshold $v_{j}$ and the discordance threshold $u_{j}$, where $v_{j} \geqslant u_{j} \geqslant p_{j} \geqslant q_{j}$. The latter is introduced because it is not necessary that the point at which preference becomes obvious coincides with the lower boundary of intolerance, as is the case with other methods of the ELECTRE type. These methods are compelled to avoid weak veto effects by taking into account only a subset of discordance indices exceeding the overall level of concordance. The influence of veto is thereby implicitly dependent on the concordance concept, and cannot be directly and distinctly controlled by the decision-maker.

The threshold model may lead to the incomparability relation which occurs when there exist at least two conflicting criteria. In this case, neither the alternative $a_{i}$ is treated to be at least as good as the profile $b$ nor the opposite. Since the profile $b$ represents the delimitation of the categories $C^{+}$and $C^{-}$, it cannot be clearly stated whether the alternative should be assigned to $C^{+}$or to $C^{-}$. Consequently, the membership of $a_{i}$ is undetermined. A solution to this problem could be the introduction of the incomparability category. The approach gives an adequate insight into the characteristics of alternatives and thus enables high adaptiveness of preferences. However, an additional class hinders the comparison and the unification of group members' choices. Besides, the incomparability category has to be an empty subset at the end of the performed analysis as it is meant to show alternatives that are neither acceptable nor unsatisfactory at a certain point in time. The decision-maker still hesitates over the status of these alternatives, but they eventually have to be unambiguously sorted. 
The localization principle thus calls for the prevention of the incomparability relation. So, the $u_{j}$ and $v_{j}$ thresholds are treated asymmetrically. This is justified by the noncompensatory nature of the veto concept and originates from the explicitly regarded primary viewpoint of the logical evaluation of the truthfulness of the alternative assignment to the positive category. This fixed point of view implicitly determines the complementary logical evaluation, which confirms or rejects the truthfulness of the assignment to the negative category. The positive semantics is mathematically denoted as: $a_{i} \in C^{+} \Rightarrow a_{i} \notin C^{-}, a_{i} \notin C^{+} \Rightarrow a_{i} \in C^{-}$.

It is only important whether an alternative is good enough to be assigned to $C^{+}$and not whether it is convenient for $C^{-}$. This is utterly reasonable, since alternatives belonging to $C^{+}$are solely chosen for further analysis or for implementation. In practice, asymmetry means that an alternative $a_{i}$ with very poor values on some criteria is excluded from $C^{+}$. It is not important though, if the profile $b$ does not reach one or more veto thresholds when compared with $a_{i}$, because this information does not confirm that $a_{i}$ is a member of the $C^{+}$class nor does it prevent the assignment of $a_{i}$ to the $C^{-}$class. Yet small weaknesses of an alternative should be compensated. Hence, the indifference and preference thresholds are treated symmetrically, thereby leading to the symmetrically-asymmetrical interpretation of preferential information.

To express the degree of concordance with the assertion "the alternative $a_{i}$ belongs to the $C^{+}$category", the indices $s_{j}\left(a_{i}, b\right)$ and $s_{j}\left(b, a_{i}\right)$ are aggregated:

$$
c_{j}\left(a_{i}\right)=\frac{1}{2} \cdot\left(s_{j}\left(a_{i}, b\right)+\left(1-s_{j}\left(b, a_{i}\right)\right)\right) .
$$

It is assured that $c_{j}\left(a_{i}\right)$ is a fuzzy averaging operator (Zimmermann, 1996) because all of its operands are defined on the $[0,1]$ interval and because the following inequality holds:

$$
\min \left(s_{j}\left(a_{i}, b\right), 1-s_{j}\left(b, a_{i}\right)\right) \leqslant c_{j}\left(a_{i}\right) \leqslant \max \left(s_{j}\left(a_{i}, b\right), 1-s_{j}\left(b, a_{i}\right)\right)
$$

For the sake of compensation of small weaknesses, the indices $c_{j}\left(a_{i}\right)$ are combined so that each is scaled by the weighting coefficient $w_{j}$ which represents the voting power of the $j$ th criterion and determines its contribution to the aggregation:

$$
c\left(a_{i}\right)=\sum_{j=1 . . n} w_{j} \cdot c_{j}\left(a_{i}\right), \quad \text { where } \sum_{j=1 . . n} w_{j}=1 .
$$

In contrast to the ELECTRE type methods and based on the proposed semantics, instead of two discordance indices, only one such index is defined for each criterion:

$$
d_{j}\left(a_{i}\right)=\max \left(\min \left(\left(g_{j}(b)-g_{j}\left(a_{i}\right)-u_{j}\right) /\left(v_{j}-u_{j}\right), 1\right), 0\right) .
$$

The indices $d_{j}\left(a_{i}\right)$ express the degree of discordance with the assertion "the alternative $a_{i}$ belongs to the $C^{+}$category". The overall non-discordance relation is grounded in 
two ways:

$$
\begin{aligned}
& \tilde{d}^{\prime}\left(a_{i}\right)=\prod_{j=1 . . n}\left(1-d_{j}\left(a_{i}\right)\right), \\
& \tilde{d}^{\prime \prime}\left(a_{i}\right)=1-d\left(a_{i}\right), \quad \text { where } d\left(a_{i}\right)=\max _{j=1 . . n} d_{j}\left(a_{i}\right) .
\end{aligned}
$$

The first non-discordance index considers the veto effects of all criteria, while the latter limits itself to a single discordance degree computed with the fuzzy union operator. The interpretation is that an alternative cannot be excluded from the positive class with greater certainty than it is excluded according to a criterion on which its performance is the poorest.

The higher the value of the $j$ th discordance index $d_{j}\left(a_{i}\right)$ is, the weaker $a_{i}$ is according to $x_{j}$, and the more its overall credibility decreases. In the case of nonadmissible deficiencies, the non-discordance index has to be low enough to exclude $a_{i}$ from the $C^{+}$category. The valued outranking relation is therefore obtained as a result of the multiplication:

$$
\sigma\left(a_{i}\right)=c\left(a_{i}\right) \cdot \tilde{d}\left(a_{i}\right), \text { so that } \tilde{d}\left(a_{i}\right)=\tilde{d}^{\prime}\left(a_{i}\right) \text { or } \tilde{d}\left(a_{i}\right)=\tilde{d}^{\prime \prime}\left(a_{i}\right)
$$

As $\sigma\left(a_{i}\right)=0.5$ denotes strict equality among the alternative and the profile, the classical $\lambda$-cut may be used to determine the "crisp" membership of the alternative:

$$
a_{i} \in C^{+} \Leftrightarrow \sigma\left(a_{i}\right) \geqslant \lambda, \text { where } \lambda \in[0.5,1]
$$

Because of the introduced positive semantics and because the index $c_{j}\left(a_{i}\right)$ combines the indices $s_{j}\left(a_{i}, b\right)$ and $s_{j}\left(b, a_{i}\right)$, there is no need to explicitly verify whether the alternative is a member of the negative category. This prevents logical nonsense, which can according to Bisdorff (2000) - occur when applying standard outranking methods. Fig. 1 gives the graphical interpretation of the criterion-wise concordance and discordance indices taking part in the logical evaluation of the assertion "the alternative $a_{i}$ belongs to the $C^{+}$category".

The outranking school has abandoned the classical framework of a preference structure based solely on the relations of preference and equality, in an attempt to be more realistic and closer to the bounded rationality of the decision-makers (Stewart and Losa, 2003). Since the nontransitive relation of incomparability no longer exists in conjunction with the presented method, appropriate metrics are defined that indicate conflicting alternatives, and help express sensible values of input parameters.

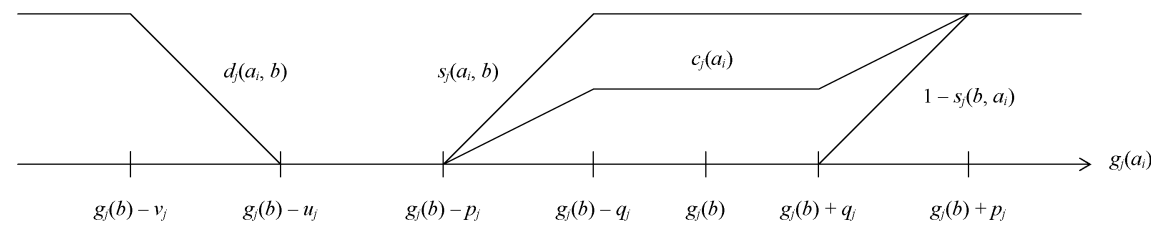

Fig. 1. The degrees of concordance and discordance with the assertion " $a_{i}$ belongs to $C^{+}$" with respect to a maximized criterion. 


\section{Robustness Metrics}

Sensitivity/robustness analysis is one of key concepts in the field of multi-criteria decision-aiding (Saltelli et al., 1999). Many approaches to it have been introduced in the past (Saltenis, 1998; Vincke, 1999). However, since they are designed for specific existing decision-making methods, an original robustness analysis technique is required to enable fully automated pseudo-criterion based group consensus seeking and multi-agent negotiation.

Three types of distance metrics are defined. They reflect the minimum changes of weight, veto and preference vectors that cause the reassignment of an alternative to the other category. When, considering the alternative $a_{i}$, any of these measures is low, the membership of $a_{i}$ is insufficiently robust, since only a slight modification of preferences may result in a different decision. Group members thus have to focus primarily on boundary alternatives and strive to clarify the reasons for their selection. Moreover, when the process is automated in an agent based setting, the interpretation of robustness degrees becomes a prerequisite to adjust input parameters in a correct manner, increase the consensus level and start a new iteration.

The most simple task is to find the smallest change of the weight vector $w=$ $\left(w_{1}, \ldots, w_{n}\right)$ so that the reassignment of an alternative to the other class occurs, that is: $a_{i} \in C_{k}^{+} \rightarrow a_{i} \in \tilde{C}_{k}^{-}$or equivalently $a_{i} \in C_{k}^{-} \rightarrow a_{i} \in \tilde{C}_{k}^{+}$. The problem is solved with an optimization program:

$$
\Delta_{w}\left(a_{i}\right)=\min \left[\sum_{j=1 . . n}\left(\left|w_{j}-\tilde{w}_{j}\right|\right)^{P} / \Delta_{w}^{\max }\right]^{1 / P} \text { by deriving } w_{j}, \quad \forall j=1, \ldots, n
$$

subject to

$$
\begin{aligned}
& \sigma\left(a_{i}\right)=d\left(a_{i}\right) \cdot\left(\sum_{j=1 . . n} w_{j} \cdot c_{j}\left(a_{i}\right)\right)=\lambda, \\
& \sum_{j=1 . . n} w_{j}=1, \quad l w_{j} \leqslant w_{j} \leqslant u w_{j}, \quad \forall j=1, \ldots, n .
\end{aligned}
$$

Here, $\tilde{w}_{j}$ are current and $w_{j}$ newly defined weights, while $l w_{j}$ and $u w_{j}$ are lower and upper bounds of allowed weight intervals. The value of the parameter $P, 1 \leqslant P \leqslant \infty$, determines which one of the $L_{P}$ distance metrics is used. The obtained distance has to be normalized by division with $\Delta_{w}^{\max }$ which denotes the largest possible change of the weight vector. For the case when all criteria weights are allowed to take any value on the interval between 0 and $1\left(\forall j: d w_{j}=u w_{j}-l w_{j}=1\right)$, the vector changes maximally when exactly two of its components move from one extreme to the other: $w_{i}=1, \forall k \neq i$ : $w_{k}=0 \rightarrow w_{j}=1, i \neq j, \forall k \neq j: w_{k}=0$. In this special situation, $\Delta_{w}^{\max }$ equals to 2 . However, for arbitrary differences $d w_{j}$, such that $\neg \forall j: d w_{j}=1$, the following mathematical program is solved:

$$
\Delta_{w}^{\max }=\max \left[\sum_{j=1 . . n}\left(\left|w_{j}^{\mathrm{end}}-w_{j}^{\mathrm{start}}\right|\right)^{P}\right]^{1 / P} \text { by deriving } w_{j}^{\text {start }}, w_{j}^{\mathrm{end}}, \forall j=1, \ldots, n,
$$


subject to

$$
\begin{aligned}
& \sum_{j=1 . . n} w_{j}^{\text {start }}=1 \\
& \sum_{j=1 . . n} w_{j}^{\text {end }}=1 \\
& l w_{j} \leqslant w_{j}^{\text {start }} \leqslant u w_{j}, \quad \forall j=1, \ldots, n, \\
& l w_{j} \leqslant w_{j}^{\text {end }} \leqslant u w_{j}, \quad \forall j=1, \ldots, n .
\end{aligned}
$$

A harder problem is to measure the robustness of discordance and veto thresholds. An advanced metric is needed that allows for the aggregation of partial discordance indices with the product operator $\tilde{d}^{\prime}\left(a_{i}\right)$, and indicates the minimal threshold changes that would cause the reassignment of the observed alternative:

$$
\Delta_{v}\left(a_{i}\right)=\min \left[\sum_{j=1 . . n}\left(\delta_{j}\right)^{P} / \sum_{j=1 . . n}\left(2 \cdot\left(g_{j}(b)-p_{j}-D_{j}^{-}\right)\right)^{P}\right]^{1 / P}
$$

by deriving $u_{j}$ and $v_{j}, \quad \forall j=1, \ldots, n$,

subject to

$$
\begin{aligned}
& \sigma\left(a_{i}\right)=c\left(a_{i}\right) \cdot \prod_{j=1 . . n}\left(1-\max \left(\min \left(\left(g_{j}(b)-g_{j}\left(a_{i}\right)-u_{j}\right) /\left(v_{j}-u_{j}\right), 1\right), 0\right)\right)=\lambda, \\
& \delta_{j}=\left|u_{j}-\tilde{u}_{j}\right|+\left|v_{j}-\tilde{v}_{j}\right|+\left|\left(v_{j}-u_{j}\right)-\left(\tilde{v}_{j}-\tilde{u}_{j}\right)\right|, \quad \forall j=1, \ldots, n, \\
& p_{j} \leqslant u_{j} \leqslant v_{j} \leqslant b_{j}, \quad \forall j=1, \ldots, n .
\end{aligned}
$$

The program minimizes the distances between new and previous values of discordance and veto thresholds. It also pays regard to the distances between different thresholds $\left(\left|v_{j}-u_{j}\right|\right)$, to prevent anomalies that can occur if thresholds converge towards the same value. The obtained distance is, again, normalized according to the least favourable situation, which appears when one threshold changes its value from one possible extreme to the other (that is from the lower bound of the criterion domain to $g_{j}(b)-p_{j}$, or the opposite), while the second remains in the starting extreme position. The presented program demonstrates the problematic of finding the smallest change of $u_{j}$ and $v_{j}$ thresholds that causes the reassignment of an alternative. Yet, it deals with piecewise linear functions with unknown segments. For this reason, it is substituted with a different optimization program. For each criterion value $g_{j}\left(a_{i}\right)$ of the alternative $a_{i}$, an appropriate partial discordance degree is found so that the product of these degrees equals the required overall discordance $\tilde{d}\left(a_{i}\right)=\lambda / c\left(a_{i}\right)$ calculated by dividing the fixed cut level $\lambda$ with the fixed concordance index. The $k_{j}$ coefficient of a linear function is then derived for each criterion $x_{j}$ according to $g_{j}\left(a_{i}\right)$ (x-axis) and $\tilde{d}_{j}\left(a_{i}\right)$ (y-axis). The induced function determines the $u_{j}$ threshold (at $y=0$ ) and the $v_{j}$ threshold (at $y=1$ ), and minimizes the distance 
metric:

$$
\Delta_{v}\left(a_{i}\right)=\min \left[\sum_{\substack{j \in F \\ \tilde{d}}}\left(\delta_{j}\right)^{P} / \sum_{j \in E}\left(2 \cdot\left(b_{j}-p_{j}-D_{j}^{-}\right)\right)^{P}\right]^{1 / P}
$$

by deriving $\tilde{d}_{j}\left(a_{i}\right)$ and $k_{j}, \quad \forall j \in F$,

subject to

$$
\begin{aligned}
& E=\{1, \ldots, n\}, \quad F \subseteq E \\
& \prod_{j \in F}\left(1-\tilde{d}_{j}\left(a_{i}\right)\right) \cdot \prod_{j \in E \backslash F}\left(1-d_{j}\left(a_{i}\right)\right)=\tilde{d}\left(a_{i}\right), \quad 0 \leqslant \tilde{d}_{j}\left(a_{i}\right) \leqslant 1, \quad \forall j \in F, \\
& \delta_{j}=\delta_{j}^{u}+\delta_{j}^{v}+\delta_{j}^{u v}, \quad \forall j \in F, \\
& \delta_{j}^{u}=u_{j}-g_{j}\left(a_{i}\right)+\tilde{d}_{j}\left(a_{i}\right) / k_{j}, \quad \forall j \in F, \\
& \delta_{j}^{v}=v_{j}-g_{j}\left(a_{i}\right)-\left(1-\tilde{d}_{j}\left(a_{i}\right)\right) / k_{j}, \quad \forall j \in F, \\
& \delta_{j}^{u v}=v_{j}-u_{j}-1 / k_{j}, \quad \forall j \in F, \\
& \left(1-\tilde{d}_{j}\left(a_{i}\right)\right) /\left(D_{j}^{+}-D_{j}^{-}-g_{j}\left(a_{i}\right)\right) \leqslant k_{j} \leqslant \tilde{d}_{j}\left(a_{i}\right) /\left(g_{j}\left(a_{i}\right)-p_{j}\right), \quad \forall j \in F .
\end{aligned}
$$

To cope with its high complexity, the problem is divided into subproblems and solved with an algorithm:

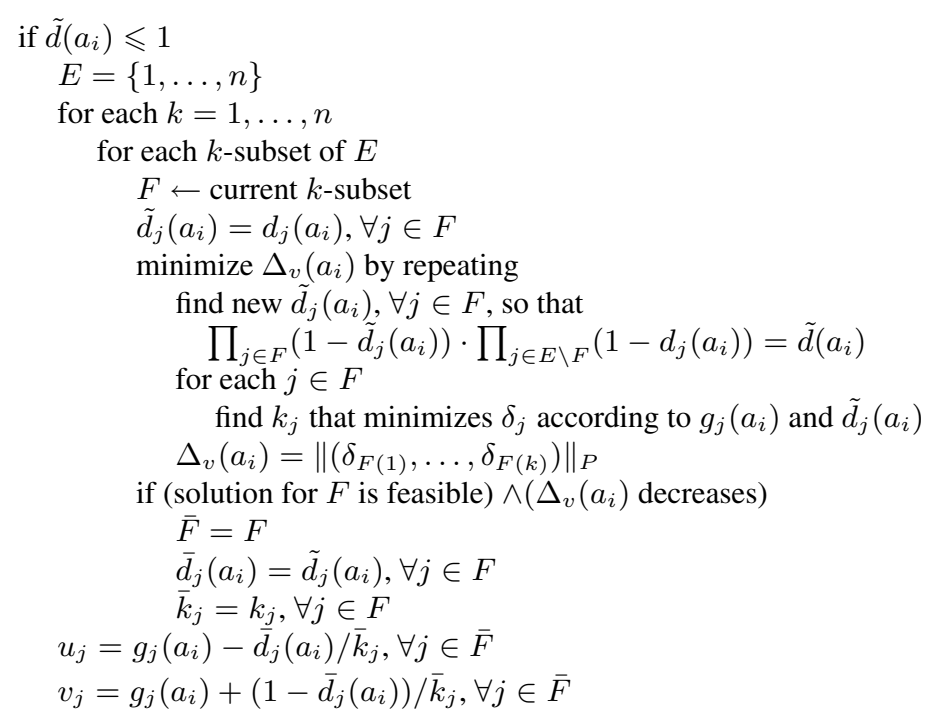

It should be noticed that is not always necessary to infer new $u_{j}$ and $v_{j}$ values for each criterion. Therefore, all possible combinations of criteria are generated and evaluated. Only the one that is feasible and yields the lowest distance is kept. If $\tilde{d}\left(a_{i}\right)>1$, the algorithm does not start, since in this case $a_{i}$ cannot be reassigned by adjusting the $u_{j}$ and $v_{j}$ thresholds in isolation.

The problem of finding the smallest changes of indifference and preference thresholds that cause the classification of an alternative into a different category is very similar to 
the one described above. The required degree of concordance is $\tilde{c}\left(a_{i}\right)=\lambda / d\left(a_{i}\right)$. The optimization is slightly more demanding because it has to deal with symmetry of partial concordance indices. This difficulty is overcome by multiplying each $\tilde{c}_{j}\left(a_{i}\right)$ index with a sign that is determined by comparing the $g_{j}\left(a_{i}\right)$ and $g_{j}(b)$ values:

$$
\Delta_{p}\left(a_{i}\right)=\min \left[\sum_{j \in F}\left(\delta_{j}\right)^{P} / \sum_{j \in E}\left(2 \cdot u_{j}\right)^{P}\right]^{1 / P} \text { by deriving } \tilde{c}_{j}\left(a_{i}\right) \text { and } k_{j}, \quad \forall j \in F
$$

subject to

$$
\begin{aligned}
& E=\{1, \ldots, n\}, \quad F \subseteq E, \\
& \frac{1}{2} \cdot \sum_{j \in F} w_{j} \cdot\left(1+\operatorname{sgn}\left(g_{j}\left(a_{i}\right)-g_{j}(b)\right) \cdot \tilde{c}_{j}\left(a_{i}\right)\right)+\sum_{j \in E \backslash F} w_{j} \cdot c_{j}\left(a_{i}\right)=\tilde{c}\left(a_{i}\right), \\
& 0 \leqslant \tilde{c}_{j}\left(a_{i}\right) \leqslant 1, \quad \forall j \in F, \\
& \delta_{j}=\delta_{j}^{q}+\delta_{j}^{p}+\delta_{j}^{q p}, \quad \forall j \in F, \\
& \delta_{j}^{q}=q_{j}-g_{j}\left(a_{i}\right)+\tilde{c}_{j}\left(a_{i}\right) / k_{j}, \quad \forall j \in F, \\
& \delta_{j}^{p}=p_{j}-g_{j}\left(a_{i}\right)-\left(1-\tilde{c}_{j}\left(a_{i}\right)\right) / k_{j}, \quad \forall j \in F, \\
& \delta_{j}^{q p}=p_{j}-q_{j}-1 / k_{j}, \quad \forall j \in F, \\
& \tilde{c}_{j}\left(a_{i}\right) / g_{j}\left(a_{i}\right) \leqslant k_{j} \leqslant\left(\tilde{c}_{j}\left(a_{i}\right)-1\right) /\left(g_{j}\left(a_{i}\right)-u_{j}\right), \quad \forall j \in F .
\end{aligned}
$$

The $\Delta_{w}\left(a_{i}\right), \Delta_{v}\left(a_{i}\right)$ and $\Delta_{p}\left(a_{i}\right)$ degrees should be aggregated with a fuzzy weighted averaging operator (Ribeiro and Marques-Pereira, 2003), because the least robust that certain parameters are, the stronger impact they have on weakening the overall robustness:

$$
r\left(a_{i}\right)=1-\left(\varpi_{(j)} \cdot \Delta_{w}\left(a_{i}\right)+\varpi_{(k)} \cdot \Delta_{v}\left(a_{i}\right)+\varpi_{(l)} \cdot \Delta_{p}\left(a_{i}\right)\right),
$$

where $j \neq k \neq l$ and $\varpi_{(\phi)}=(4-\phi) / \sum_{\varphi=1}^{3} \varphi$.

\section{Process of Group Decision-Making}

\subsection{Compromise, Consensus and Agreement Degrees}

To direct the process of group decision-making, in the sense of preference unification, the consensus and agreement measures are defined. These measures are similar to the ones that have been proposed by Herrera-Viedma et al. (2002). Their method, however, relies on direct specification of fuzzy preferential relations and combines individuals' solutions by applying an ordered weighted geometric operator (Herrera et al., 2001). The presented method, on the contrary, implements the pseudo-criterion concept to elicit input information and does not force an aggregated rank-order of alternatives on the decisionmakers. 
A compromise is ensured in a very simple way. An acceptable alternative is assigned to the positive category. It thereby receives one vote. As all group members operate on the same alternative set, votes are plainly added. Let $o$ be the number of decision-makers and $C_{k}^{+}$the subset of alternatives that are approved by the $k$ th individual. Then the sum of votes for $a_{i}$ is

$$
v_{i}=\operatorname{card}\left(a_{i} \in C_{k}^{+}, \quad k=1, \ldots, o\right)
$$

Alternatives can now be ranked from the most preferable ones, for which $v=$ $\max _{i=1 . . m} v_{i}$ holds true, to those that receive the least votes. It is thus clear how many participants in the decision-making process agree upon a given choice and it can never happen that a decision is made, which is not in accordance with the opinion of the majority of people involved.

Since a high level of comparability is attained as a consequence of the applied localization principle, it is an uncomplicated task to define the consensus measure. Let $z_{i}$ be the consensus degree reached for the alternative $a_{i}$. The equality $z_{i}=0$ holds true, if half of individuals in the group assign $a_{i}$ to the $C^{+}$category and the other half to the $C^{-}$category. The greatest possible separateness between decision-makers occurs in this case, so it cannot be determined whether $a_{i}$ is an appropriate choice. On the contrary, $z_{i}$ equals to 1 when all participants classify $a_{i}$ into the same class, thereby making the group totally uniform. Let $\nu_{i}^{+}=v_{i}$ and $\nu_{i}^{-}=o-v_{i}$ denote how many participants assign $a_{i}$ to the $C^{+}$class and to the $C^{-}$class, respectively. Then

$$
z_{i}=\frac{\nu_{i}-\rho}{o-\rho}, \quad \text { where } \nu_{i}=\max \left(\nu_{i}^{+}, \nu_{i}^{-}\right)
$$

Another measure is important for the sake of active preference unification. It is called the agreement degree. If the $k$ th decision-maker assigns $a_{i}$ to the same class as all the other group members, then he agrees with the majority opinion. Thus, $\zeta_{i}^{k}=1$. On the contrary, $\zeta_{i}^{k}=0$, if according to his preferential parameters, $a_{i}$ belongs to the category that is in opposition to the collective choice. So, the more people that assign $a_{i}$ to the same category as an individual does, the higher the level of agreement that is reached from the perspective of this person:

$$
\zeta_{i}^{k}= \begin{cases}\left(\nu_{i}^{+}-1\right) /(o-1), & a_{i} \in C_{k}^{+} \\ \left(\nu_{i}^{-}-1\right) /(o-1), & a_{i} \in C_{k}^{-}\end{cases}
$$

An operator which aggregates the partial consensus indices (and similarly, the partial agreement indices), should not only ensure compensation but has to consider the weakest alternative as well. For this reason, Werners' fuzzy "and" (Zimmermann, 1996) is chosen:

$$
Z=\gamma \cdot \min _{i=1 . . m} z_{i}+(1-\gamma) \cdot \frac{\sum_{i=1 . . m} z_{i}}{m}, \quad \gamma \in[0,1]
$$

Many researchers and practitioners - like for instance Jabeur et al. (2004), LeyvaLópez and Fernández-González (2003), or Zhang and Lu (2003) - state that in real-world 
decision groups, it is often the case that certain actors may be considered as playing a "leading" role, because they hold the responsibility and the possibility for realizing the chosen solution, have recognized abilities and interests for specific problems, or occupy privileged positions. To enable a hierarchical decision-making situation, group members are given importance weights $\omega_{k}, k=1, \ldots, o$, and the degrees of compromise, consensus and agreement are anewly defined:

$$
\begin{aligned}
& v_{i}= \frac{\sum_{k \in E} \omega_{k}}{\sum_{k=1 . . o} \omega_{k}}, \text { where } E=\left\{\forall k=1, \ldots, o: a_{i} \in C_{k}^{+}\right\}, \\
& z_{i}=2 \cdot\left(\nu_{i}-1 / 2\right), \text { where } \nu_{i}=\max \left(v_{i}, 1-v_{i}\right), \\
& \zeta_{i}^{k}=\frac{\omega_{k}+\sum_{l \in F} \omega_{l}}{\sum_{l=1 \ldots o} \omega_{l}}, \\
& \quad \text { where } F=\left\{\begin{array}{l}
\forall l=1, \ldots, o, l \neq k: \\
\left(a_{i} \in C_{k}^{+} \wedge a_{i} \in C_{l}^{+}\right) \vee\left(a_{i} \in C_{k}^{-} \wedge a_{i} \in C_{l}^{-}\right)
\end{array}\right\} .
\end{aligned}
$$

The only difference that should be noticed in comparison with the previous "democratic" definitions regards to the interpretation of the agreement degree. It is always higher than 0 , because it is presupposed that a decision-maker agrees with himself. This is relevant when an autocratic individual is present in the group and when he is in contradiction with other team members. The decision support system must not demand from such a decision-maker to be the first to conform to the opinions of colleagues. It is obvious, though, that if the weight of this person would not be considered, exactly this kind of a situation would occur.

\subsection{Mechanism of Consensus Seeking}

The active mechanism of directing group members toward unified opinions is founded on the progressive increasing of the consensus degree $Z$ toward the specified threshold $\xi$. At first sight, it seems that $z_{i}$ has to be primarily raised for those alternatives which have reached a low degree of consensus $\left(z_{i} \approx 0\right)$. Their status is namely absolutely undetermined. Neither can they be with certainty assigned to the positive nor to the negative category since there does not exist a prevailing majority of decision-makers that would have enough strength to approve or disprove the suitability of their selection. But such indetermination disables the decision support system to effectively advise a person about the adjustment of preferential parameters. In the case when exactly half of decision-makers assign $a_{i}$ to $C^{+}$and another half to $C^{-}$, it is not evident in which direction the category change should be carried out. Hence, the problem of reaching a consensus is approached from the other side. It is presumed that it is the most credible task to increase the degree of consensus for alternatives that already have a high $z_{i}$ value. If it is close to $1\left(z_{i} \approx 1\right.$, but compulsorily $z_{i}<1$ ), two facts may be taken into account:

- It is clear toward which category the group opinion leans when evaluating an alternative with a high level of consensus. Thus, it is righteous to demand the category change from the individuals that oppose this opinion as they are in the absolute, uninfluential minority. 
- At first, the group concentrates on alternatives that are rather uniformly judged by its members. A full agreement about very good alternatives can therefore quickly be found. As these alternatives generally suffice for the right decision, other alternatives need not be dealt with at all or can be left for later consideration.

The outline of the consensus seeking procedure is presented on Fig. 2. The decisonmaker with the lowest degree of agreement is selected in each iteration. Since this individual is in the strongest opposition to the collective choice, his preferential attitude is the principal reason why the value of $Z$ is not high enough. He has to adjust the values of input parameters to such an extent that someone else becomes the most contradictive group member. Since it is always the turn of the participant with the lowest computed agreement level, two gains arise:

- the values of $\zeta^{k}$ incessantly increase ensuring the convergence of $Z$ toward the threshold $\xi$;

- equality among decision-makers can be guaranteed, as the only measure of the required conformation to the opinions of colleagues is the deviation from the collective choice, which is independent of the person's rank, except when hierarchical metrics are applied.

It is reasonable that the decision-maker reassigns only alternatives with a low value of the agreement index and with a low robustness level. Otherwise, either a satisfactory agreement degree is reached from this person's perspective, or his opinion is so firmly stated that the conformation to the group is not sensible in spite of a considerable contradiction with it. It is thus the obligation of the decision support system to show for each alternative its partial agreement index as well as data on its sensitivity. The obtained information enables manual selection of alternatives which are subject to the reassignment. This is essential because the decision-maker must be able to reject the proposed category changes. When he is convinced that his judgment is right, he may insist on his own choice. Other participants are thereby stimulated to rethink about the decision, en-

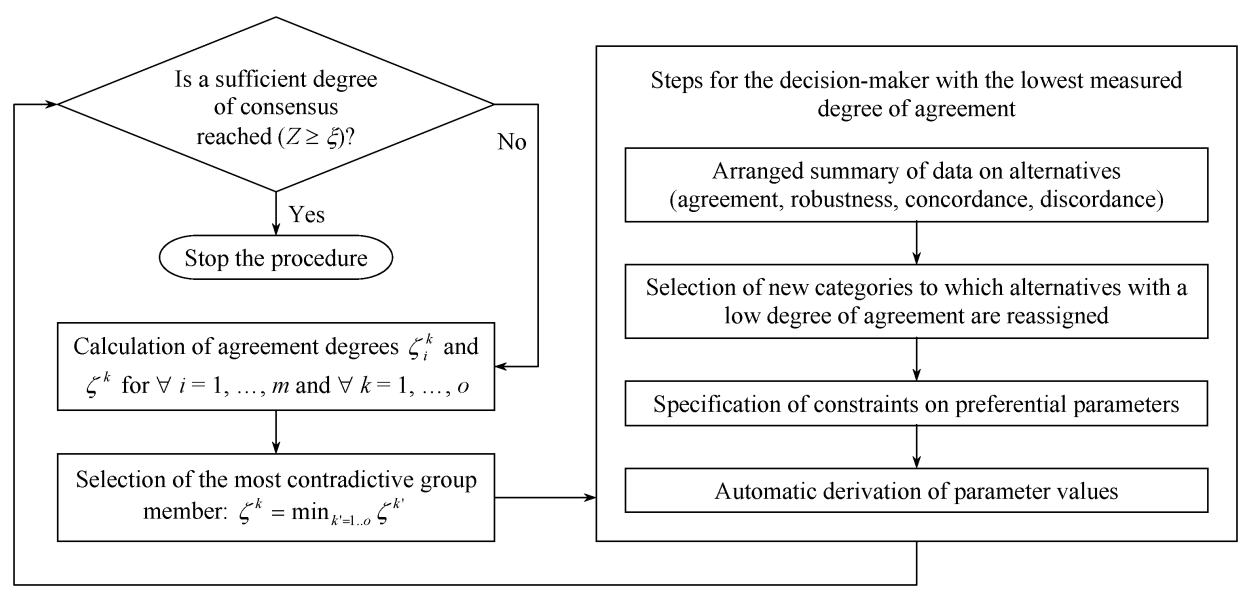

Fig. 2. The group consensus seeking procedure. 
lighten their understanding of the problem from complementary viewpoints, and consider important facts that they have perhaps overlooked.

Suppose the decision-maker states which conflicting alternatives he is prepared to reassign: $a_{i} \in C_{k}^{+} \rightarrow a_{i} \in \tilde{C}_{k}^{-}$or $a_{i} \in C_{k}^{-} \rightarrow a_{i} \in \tilde{C}_{k}^{+}$. New parameter values can then be automatically derived so that the required changes are attained for the chosen alternatives and so that other memberships are preserved. Parameters are inferred by solving an optimization program:

$$
\operatorname{maximize} \min \left\{\tau_{i}^{+}, \tau_{i}^{-}\right\}_{i=1 . . m}
$$

subject to

$$
\begin{aligned}
& \sigma\left(a_{i}\right)-\tau_{i}^{+}=\lambda, \quad \forall a_{i} \in \tilde{C}_{k}^{+}, \\
& \sigma\left(a_{i}\right)+\tau_{i}^{-}=\lambda, \quad \forall a_{i} \in \tilde{C}_{k}^{-}, \\
& 0 \leqslant q_{j} \leqslant p_{j} \leqslant u_{j} \leqslant v_{j} \leqslant b_{j}-D_{j}^{-}, \quad \forall j=1, \ldots, n, \\
& l w_{j} \leqslant w_{j} \leqslant u w_{j}, \quad \forall j=1, \ldots, n, \\
& \sum_{j=1 . . n} w_{j}=1, \\
& \lambda \in[0.5,1] .
\end{aligned}
$$

The category membership changes at the value of $\sigma\left(a_{i}\right)=\lambda$. The variables $\tau_{i}^{+}$and $\tau_{i}^{-}$must be positive to ensure the assignment of an alternative to the proper category. The lowest of them is maximized by the optimization program for the purpose of achieving robustness. To acquire credible results, it is sensible that the decision-maker specifies additional constraints, for example intervals of suitable criteria weights. In this way, the modification of parameters that are in accordance with the individual's preferences is prevented.

\subsection{Automated Cooperative Agent Based Negotiation}

It is essential for an efficient group decision-making procedure that the cognitive burden of each individual decision-maker as well as the human moderator is reduced to the minimum. Since in certain problem solving situations the latter is potentially biased in favour of its own opinion or against the judgements of some group members, it is advisable that its activity is eliminated. The implemented system must hence be able to autonomously guide the problem solving process. Moreover, people are not always willing to directly engage into synchronous or asynchronous communication. There exist many types of problems and e-services in the context of which they express initial preferences and expect the information system to find the optimal available solution by representing their interests (Bichler et al., 2003; Lomuscio et al., 2003). It is thus crucial to enable decisionmaking and negotiation in an agent based setting.

The centralized agent negotiation architecture is modelled on Fig. 3. The central role is given to the analytical and mediation agent. It is responsible for preference aggregation and alternative sorting on the individual level; calculation of compromise, consensus, 


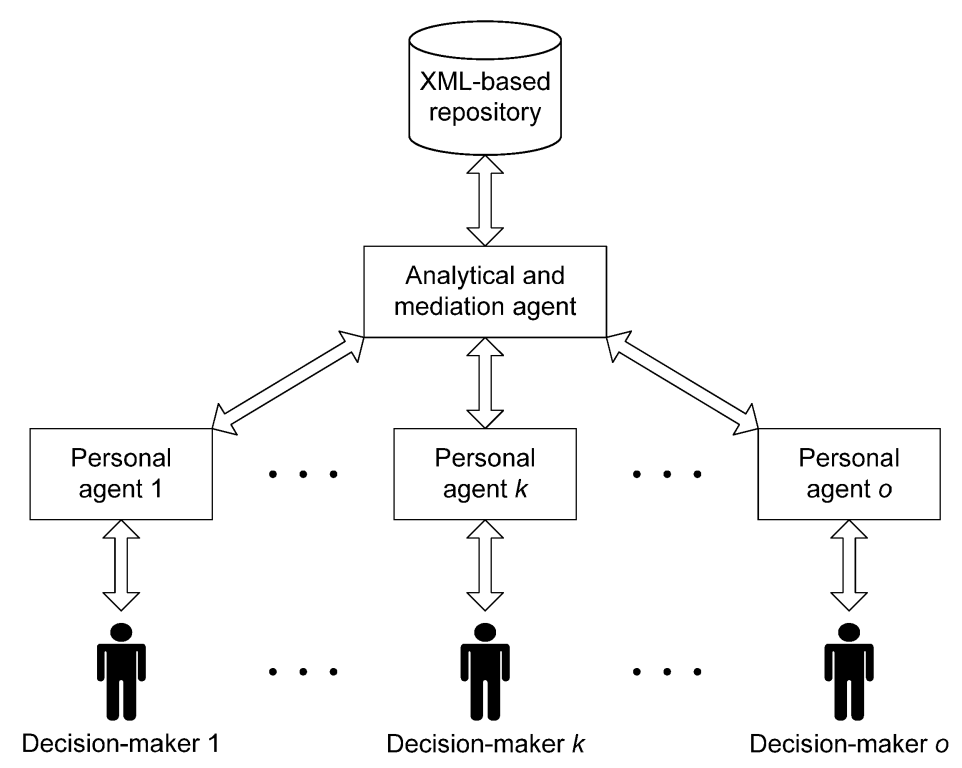

Fig. 3. Agent negotiation architecture.

agreement and robustness degrees; automatic adjustment of preferential parameters; negotiation process directing; and accessing the XML based repository. It communicates with personal agents that are not mutually connected. Each of them corresponds to a single decision-maker from whom it receives requests and preferential information, and in the name of whom it negotiates.

The defined centralized architecture has several advantages. It simplifies the collaboration between the agents because it eliminates the need for each one of them to inform all the others about its activities. The number of required interactions is therefore considerably decreased. In addition, only the mediation/analytical agent has to implement the decision-making logic with optimization facilities, and must provide support for the XML based repository. Personal agents are consequently regarded as thin clients. They merely offer the user interface to the decision-maker and ensure that his expectations are met.

In some cases, time constraints on synchronous preference aggregation/disaggregation, and in particular optimization algorithms, may be an issue (Dzemyda and Petkus, 2001). Then, the computational load is distributed through the network by transmitting it to all agents. Each personal agent has the responsibility to sort alternatives, compute the robustness degrees and infer parameters for the decision-maker that it represents. However, such agents cannot be implemented as thin clients, but rather as .NET or Java web services capable of executing complex optimizations.

As the agents strive to reach consensus, their behaviour tends to be cooperative, providing benefits to all involved parties and assuring an agreement on a commonly accepted solution. The protocol of agent based negotiation extends the consensus seeking mechanism for group decision-making. Its definition is given by means of an algorithm in 
which $\mathrm{DM}_{k}$ denotes the $k$ th decision-maker, $\mathrm{PA}_{k}$ the $k$ th personal agent and MAA the mediation/analytical agent.

for each $k=1, \ldots, o$

$\mathrm{DM}_{k}$ specifies initial values of preferential parameters and their permissible deviations

$\mathrm{PA}_{k}$ transmits initial parameter values to MAA repeat

MAA sorts alternatives for each $\mathrm{PA}_{k}$ into the categories $C_{k}^{+}$and $C_{k}^{-}$

MAA calculates the agreement, consensus and robustness degrees $\zeta_{i}^{k}, \zeta^{k}, z_{i}, Z$ and $r_{i}^{k}$

if $Z<\xi$

MAA sorts $\mathrm{PA}_{k}$ in the ascending order so that $\mathrm{PA}_{(l)}$ exhibits the $l$ th lowest $\zeta^{k}$ degree conformation $\leftarrow$ false

while $l \leqslant o$ and conformation $=$ false

reassignment $\leftarrow$ false

for each $i=1, \ldots, m$

if $\zeta_{i}^{l}<\frac{1}{2}$ and $r_{i}^{l}<\psi$

MAA reassigns $a_{i}$ so that $a_{i} \in C_{l}^{+} \rightarrow a_{i} \in \widetilde{C}_{l}^{-}$or $a_{i} \in C_{l}^{-} \rightarrow a_{i} \in \widetilde{C}_{l}^{+}$

reassignment $\leftarrow$ true

if reassignment $=$ true

MAA inferres robust values of preferential parameters according to $\widetilde{C}_{l}^{+}$and $\widetilde{C}_{l}^{-}$

MAA transmits inferred parameter values and initial/new assignments to $\mathrm{PA}_{(l)}$

if permissible deviations of $\mathrm{PA}_{(l)}$ are not violated

conformation $\leftarrow$ true

else // optional

$\mathrm{DM}_{(l)}$ reconsiders parameter values and allowed deviations

if $\mathrm{DM}_{(l)}$ accepts required adjustments

conformation $\leftarrow$ true

$\mathrm{PA}_{(l)}$ transmits new parameter values to MAA

if conformation $=$ false

$l \leftarrow l+1$

if $l>o$ and $\exists l^{\prime}=\min l$ : reassignment $=$ true

$\mathrm{PA}_{\left(l^{\prime}\right)}$ is forced to conformation by adjusting parameter values and/or deviations

until $Z \geqslant \xi$ or $\forall k=1, \ldots, o$ : reassignment $=$ false

if $Z<\xi$

alternatives are ranked according to the degrees of compromise $v_{i}$ and robustness $r_{i}$

The mediation/analytical agent eliminates the need for a human moderator. Moreover, the cognitive load and the activity of each decision-maker are minimized. His only mandatory task is to specify initial values of preferential parameters and their permissible deviations that may not be exceeded by the mediation/analytical agent in the process of parameter inferrence. It is possible to provide this information in several forms:

- by specifying approximate linguistic modifiers of predetermined threshold magnitudes;

- by setting the exact initial values of parameters $b_{j}, q_{j}, p_{j}, u_{j}, v_{j}$ and $w_{j}$, and the exact lower respectively upper bounds $b_{j}^{-}, b_{j}^{+}, q_{j}^{-}, q_{j}^{+}, p_{j}^{-}, p_{j}^{+}, u_{j}^{-}, u_{j}^{+}, v_{j}^{-}, v_{j}^{+}$, $w_{j}^{-}$and $w_{j}^{+}$of parameters;

- by determining only the exact lower and upper bounds of allowed parameter intervals, in which case the central points of intervals are taken by the $k$ th personal agent as the initial values $b_{j}, q_{j}, p_{j}, u_{j}, v_{j}$ and $w_{j}$. 
In the first case, the magnitudes of $q_{j}, p_{j}, u_{j}$ and $v_{j}$ thresholds are expressed with decibels (Rogers and Bruen, 1998a). Since the decibel is a dimensionless quantity without physical units, it is appropriate for criteria modelling within a wide range of decision problems. It can prevent subjectiveness in judgement, and thereby also potentialy irrational comprehension of imprecision and uncertainty. It determines the relationship between a variable $S$ and a known reference $S_{0}$ by calculating the logarithm of their ratio:

$$
\mathrm{dB}=10 \cdot \log _{10}\left(S / S_{0}\right)
$$

By extending the propositions of Rogers and Bruen, the magnitudes are predetermined in the following manner:

$$
q_{j}=\Omega \cdot 2 \mathrm{~dB}, \quad p_{j}=\Omega \cdot 5 \mathrm{~dB}, \quad u_{j}=\Omega \cdot 10 \mathrm{~dB} \text { and } v_{j}=\Omega \cdot 15 \mathrm{~dB},
$$

where $\Omega \in\{0.2,0.4,0.6,0.8,1\}$ is a numerical modifier obtained with a direct transformation of a linguistic influence level $\varsigma \in$ \{very weak, weak, moderate, strong, very strong\}. Different degrees of influence may be chosen for various thresholds by the decision-maker. He has to specify the initial modifiers $\Omega_{j}$ as well as the lower and upper bounds $\Omega_{j}^{-}$and $\Omega_{j}^{+}$. Based on these values, the initial, minimal and maximal threshold magnitudes are computed by the $k$ th personal agent relative to the reference profiles $b_{j}$. The $j$ th indifference threshold is set to

$$
q_{j}=b_{j} \cdot\left(\mathrm{e}^{Q}-1\right), \quad \text { where } Q=\frac{2 \Omega_{j}}{10 \log _{10} \mathrm{e}} .
$$

Analogously, the magnitudes of $q_{j}^{-}, q_{j}^{+}, p_{j}, p_{j}^{-}, p_{j}^{+}, u_{j}, u_{j}^{-}, u_{j}^{+}, v_{j}, v_{j}^{-}$and $v_{j}^{+}$are calculated. It is the role of the $k$ th personal agent to ensure that the $k$ th decision-maker's requirements are fulfiled. The latter is given the opportunity to revise his preferences in each iteration of the negotiation process. However, he is not unconditionally obliged to that, since the negotiation mechanism is enabled to proceed without any human interaction. Once the decision-makers' initial preferences are set, the agents can autonomously collaborate, and automatically reach a common decision.

An agent is asked to conform to the others with regard to the alternative $a_{i}$ only if $a_{i}$ is not robustly sorted and if its evaluation contradicts judgments of more than half agents. In order to determine the sensitivity of assignments, the $\psi$ threshold is introduced. The alternative $a_{i}$ is hence robustly sorted into the $C^{+} / C^{-}$category when its $r\left(a_{i}\right)$ degree exceeds the $\psi$ threshold.

It is reasonable that the most contradictive personal agent, exhibiting the lowest $\zeta^{k}$ degree, is subjected to conformation. However, if all of its assignments are robust or if the adjusted parameter values violate the $k$ th decision-maker's constraints, it may be skipped, and the next most discordant personal agent may be chosen to negotiate. It is possible that the analytical and mediation agent must address several personal agents to find the one which is willing to accept the proposed changes. In the worst case, unsuccessful iteration 
over all personal agents causes the negotiation process to terminate without reaching a consensus. A compromise is then made by ranking alternatives in the descending order according to their $v_{i}$ levels. When a tie occurs, which means that $v_{i}=\ldots=v_{j}$ for two or more alternatives, it is resolved based on the multi-agent robustness degrees $\Gamma_{i}$. The higher the $\Gamma_{i}$ degree is, the better $a_{i}$ is. So:

$$
\begin{aligned}
& a_{i} \succ a_{j} \Leftrightarrow\left(v_{i}>v_{j}\right) \vee\left(\left(v_{i}=v_{j}\right) \wedge\left(\Gamma_{i}>\Gamma_{j}\right)\right), \\
& a_{i} \approx a_{j} \Leftrightarrow\left(v_{i}=v_{j}\right) \wedge\left(\Gamma_{i}>\Gamma_{j}\right),
\end{aligned}
$$

where $\succ$ and $\approx$ denote the relations of preference and indifference, respectively. $\Gamma_{i}$ is obtained as the difference between the positive and negative robustness degrees:

$$
\Gamma_{i}=\left|\Gamma_{i}^{+}-\Gamma_{i}^{-}\right|
$$

so that

$$
\Gamma_{i}^{+}=\frac{\sum_{k \in E} r_{i}^{k}}{o}, \quad \text { where } E=\left\{\forall k=1, \ldots, o: a_{i} \in C_{k}^{+}\right\}
$$

and

$$
\Gamma_{i}^{-}=\frac{\sum_{k \in F} r_{i}^{k}}{o}, \quad \text { where } F=\left\{\forall k=1, \ldots, o: a_{i} \in C_{k}^{-}\right\}
$$

Each personal agent communicates with the mediation/analytical agent via XML based messages, which is a standard approach to invoke web services, and to interchange decision models (Kim, 2001). Because of the compactness of presentation, the data structures of these messages are defined in the DTD notation (W3C, 2006). Personal agents transmit preferential parameters of decision-makers in the PA_parameters data structure to the mediation/analytical agent, while the latter transmits inferred parameter values and original/new sorting results in the opposite direction by using the MAA_parameters_assignments root element.

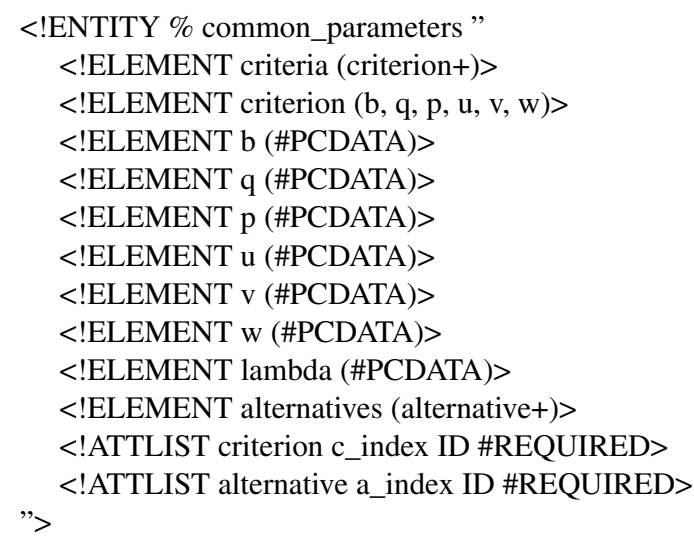




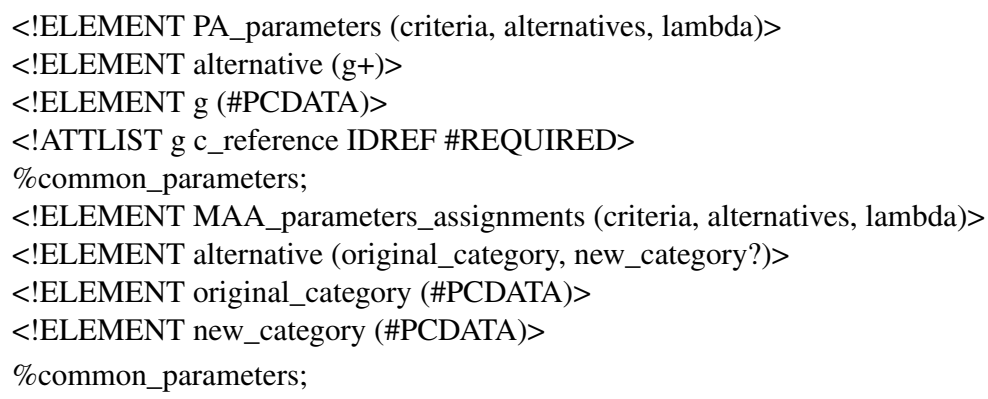

The mediation/analytical agent can access the XML repository in which several aspects of decision models are stored: general data on personal agents and/or decisionmakers; criteria-wise values of alternatives and preferential parameters for each personal agent and for each iteration; classifications of alternatives for each personal agent and for each iteration, together with corresponding measures of compromise, consensus, agreement and robustness; and the final choice with its perceived real-life effectiveness evaluated after its implementation.

Historical data about problem solving processes can be used to extract deep organizational knowledge in all subsequent group decision-making or negotiation situations. Each current problem setting can be compared with existing ones by utilizing fuzzy measures of similarity. In the case when similarity reaches the specified cut-level, the consensus seeking mechanism is able to derive reliable conclusions and suggest the optimal feasible decision without going through the process of preference unification at all, or by performing just a small subset of otherwise required iterations. This mechanism represents an independent research topic.

\section{Derivation of Criteria Weights}

The weight derivation procedure consists of the following steps:

1. A fuzzy veto relation $V=\left\{\left(\left(x_{j}, a_{i}\right), \mu_{V}\left(x_{j}, a_{i}\right)\right) \mid\left(x_{j}, a_{i}\right) \in \mathrm{X} \times \mathrm{A}\right\}$ is constructed by organizing partial discordance indices so that $\mu_{V}\left(x_{j}, a_{i}\right)=d_{j}\left(a_{i}\right)$, where $i=$ $1, \ldots, m, j=1, \ldots, n$.

2. Partial selective strengths of criteria are calculated for each $\alpha$-cut of the fuzzy relation $V$.

3. Partial selective strengths are consecutively joint by the use of an algorithm, which considers veto certainty and similarities between intermediate results.

4. Differences between complete selective strengths are transformed so that ratios of criteria weights are reflected through a pairwise comparison matrix.

5. The decision-maker modifies ratios in the comparison matrix according to his preferences.

6. Numerical values of weights are computed from the adjusted matrix.

All $\alpha$-cuts of the fuzzy veto relation $V$ are taken. The fuzzy weight derivation problem is thereby transformed into many corresponding subproblems formulated in the classical 
"crisp" sense. Obeying a heuristic rule, selective strengths of criteria are calculated for each crisp relation. The total selective strength of the $j$ th criterion is computed according to the number of alternatives that are excluded from the positive category $C^{+}$because of the discordance effect of the discordance and veto thresholds $u_{j}$ and $v_{j}$, and simultaneously according to the number of other criteria from the set $X \backslash\left\{x_{j}\right\}$ that oppose a veto on the assignment of the same alternatives to the $C^{+}$class. The partial selective strength of the criterion $x_{j}$ considers only the $i$ th alternative and the single cut-level $\alpha_{k}$. It equals to zero when $u_{j}$ and $v_{j}$ do not contradict the assertion $a_{i} \in C^{+}$or when all criteria oppose a veto on this assertion. It indicates to which degree a criterion outperforms the weakest criterion:

$$
\varphi_{j i}^{k}= \begin{cases}\operatorname{card}\left(x_{l} \in X \backslash\left\{x_{j}\right\}: d_{l}\left(a_{i}\right)<\alpha_{k}\right), & d_{j}\left(a_{i}\right) \geqslant \alpha_{k}, \\ 0, & d_{j}\left(a_{i}\right)<\alpha_{k}\end{cases}
$$

It can be stated that the partial selective strength of the $j$ th criterion, which excludes the $i$ th alternative from the positive category, equals the number of criteria, which do not exclude the same alternative. The algorithm that joins the $\varphi_{j i}^{k}$ indices rests on the following principles:

- The criterion $x_{j}$ gains the highest possible selective strength, measured according to the alternative $a_{i}$, at the first cut for which the discordance degree $d_{j}\left(a_{i}\right)$ exceeds the $\alpha_{k}$ threshold. At levels $\alpha_{k^{\prime}}>\alpha_{k}$, the indices equal to zero and need not be dealt with, while at $\alpha_{k^{\prime}}<\alpha_{k}$, additional criteria might oppose a veto and thus $\varphi_{j i}^{k^{\prime}} \leqslant \varphi_{j i}^{k}$ holds true.

- When $\varphi_{j i}^{k_{1}}=\ldots=\varphi_{j i}^{k_{h}}$ for adjacent $\alpha_{k_{1}}>\ldots>\alpha_{k_{h}}$, only the cut with the highest level is considered. This originates from the logical maximum concept: the highest certainty with which the partial strength $\varphi_{j i}^{k}\left(k=k_{1}, \ldots, k_{h}\right)$ affects the total strength $\Phi_{j}$ equals $\alpha_{k_{1}}$, so the lower certainties $\alpha \in\left[\alpha_{k_{h}}, \alpha_{k_{1}}\right)$ cannot intensify the influence of $\varphi_{j i}^{k}$.

- When the difference $\delta=\varphi_{j i}^{k}-\varphi_{j i}^{k^{\prime}}$ exceeds 0 for $\alpha_{k^{\prime}}<\alpha_{k}$, the total strength of $x_{j}$ according to $a_{i}$ falls by $\alpha_{k^{\prime}} \cdot \delta$. The difference $\delta$ has to be lessened by the certainty factor of results. The decrease is a consequence of a weaker veto effect of one or more additional critera.

- Cut-levels $\alpha_{k}, k=1, \ldots, l$, are treated as weights. The higher the cut-level is, the more certain is the crisp veto relation. Selective strengths of criteria, which are bound to cuts with levels $\alpha_{k}>>0$, are consequently more substantial for a given problem situation than those, which correspond to low levels $\alpha_{k} \approx 0$. They are entitled to contribute to a greater extent to the total strengths.

The simple algorithm that computes total selective strengths $\Phi_{j}$ is written in pseudocode. It holds: $\forall i, j=1, \ldots, l: i<j \Rightarrow \alpha_{i}>\alpha_{j}$.

$$
\begin{aligned}
& \phi_{j i}=0, \varphi_{j i}^{0}=0 \text { for } \forall i=1, \ldots, m, \forall j=1, \ldots, n \\
& \forall k=1, \ldots, l \\
& \quad \forall i=1, \ldots, m, \forall j=1, \ldots, n \\
& \quad \delta=\varphi_{j i}^{k-1}-\varphi_{j i}^{k}
\end{aligned}
$$




$$
\begin{aligned}
& \text { if } \delta \neq 0, \text { then } \phi_{j i}=\phi_{j i}-\alpha_{k} \cdot \delta \\
& \Phi_{j}=\sum_{i=1 . . m} \phi_{j i}
\end{aligned}
$$

The $\Phi_{j}$ strengths are of great help to the decision-maker when specifying criteria weights, as they reflect the relationships between criteria importances. In addition, when the value of $\Phi_{j}$ is too high or too low, the domination or the discrimination of the $j$ th criterion is exposed. This is a clear sign for the decision-maker to modify input parameters if he wishes that all criteria are adequately participating in the evaluation process.

Selective strengths must, however, not be directly interpreted as weights. The meaning of the "zero strength" has to be considered. If $\Phi_{j}=0$, it does not imply that the weight $w_{j}$ also equals to 0 , since the inability of the criterion $x_{j}$ to veto the assignment of any alternative to the positive class should not prevent its participation in the concordance test. Similarly, the maximal possible strength $\Phi_{\max }=m \cdot(n-1)$, which appears when a single criterion eliminates all alternatives from the $C^{+}$class, has to be dealt with. And finally, computed strengths should be modified by the individual in order to properly match with his personal beliefs.

Zeleny (1982) has written about criteria in the sense of information sources. Each criterion weight is linked with inner information, which is generated by the set of alternatives, and with the decision-maker's subjective assessment of importance that is bound to his experience and knowledge. The concept of importance that is dependent on the internal information flows is hence represented by the selective strength, as it is sensitive to the chosen values of the $u_{j}$ and $v_{j}$ thresholds as well as to the changes in the set of alternatives. Since this implicit information must be enriched with explicitly provided input data, two interrelated problems arise:

1. the conversion of indices $\Phi_{j}$ to weights $w_{j}$, for $j=1, \ldots, n$,

2. the representation of indices $\Phi_{j}$ in such a way that criteria importances are correctly and intelligibly expressed, and that the decision-maker is able to easily adjust them.

To solve both problems, the computed selective strengths may be transformed so that the relations between criteria are expressed in the notation of one of the available structured weight derivation techniques. Figueira and Roy (2002) believe that the Simos' procedure is the only one appropriate to be applied in conjunction with the pseudo-criterion based decision models, while Macharis et al. (2003) suggest the use of AHP matrices for the purpose of enhancing PROMETHEE through an additional weight determination mechanism.

The differences $\Delta_{i j}$ of each two selective strengths are thus transformed to be included in a $n \times n$ pairwise comparison matrix. This matrix contains ratios of criteria weights and is consistent with the concepts of AHP (Saaty, 1980) - it is reflexive, reciprocal and limited to the scale of 1 to 9 . Because of the above illustrated " $\Phi_{\max }$ problem" and because the $\Phi_{i} / \Phi_{j}$ ratio can be computed only if $\Phi_{j} \neq 0$, an approach is used that combines ratios with intervals.

Let the weight ratio be denoted as $r_{i j} \approx w_{i} / w_{j}$. The fundamental presumption is that $r_{i j}$ increases linearly according to the difference $\Delta_{i j}=\Phi_{i}-\Phi_{j}$ between the selective strengths of two criteria. The ratio $r_{i j}$ remains the same for all feasible values of $\Phi_{i}$ and $\Phi_{j}$, if only the difference $\Delta_{i j}$ is constant. The interpretation is that the additional 
strength $d \Phi_{j}$, which is gained by the criterion $x_{j}$, influences the weight increase with equal intensity regardless of the initial value $\Phi_{j}$. Since $\Phi_{\max }$ has the maximum possible priority over $\Phi_{\min }=0$, it is evident that $r_{\max }=9$ is assigned to $\Delta_{\max }=\Phi_{\max }-\Phi_{\min }$. Thus

$$
r_{i j}=\frac{8}{\Delta_{\max }} \cdot \Delta_{i j}+1
$$

Only non-negative differences are considered; if $\Delta_{i j}<0$, a reciprocal value $r_{i j}=$ $1 / r_{j i}$ is taken. The constant $b=1$ ensures that $\Delta_{i j}=0$ is transformed to the $r_{i j}=1$ ratio which indicates total equality of criteria. The linear function does not guarantee matrix consistency, so the exponential function is also defined:

$$
r_{i j}=\left(r_{\max }\right)^{E}, \text { where } E=\frac{\Delta_{i j}}{\Delta_{\max }},
$$

such that $r_{i j}=1$, if $\Delta_{i j}=0 ; r_{i j}=r_{\max }=9$, if $\Delta_{i j}=\Delta_{\max }$; and $r_{i k}=r_{i j} \cdot r_{j k}$ for $\Delta_{i k}=\Delta_{i j}+\Delta_{j k}$

Experiments show that the inconsistency rates of pairwise matrices, which are constructed with the linear function, are low. They do not rise above the worst acceptable level of 0.1 . The average value obtained for various numbers of alternatives and criteria is 0.01 .

\section{Conclusion}

In this paper, the two-categorical alternative sorting analysis based on the pseudocriterion concept was introduced. To enable this kind of analysis, the asymmetrical treatment of veto and discordance thresholds was grounded and applied. As a consequence of the localization principle, high adaptability of the quantitative decision model and high comparability of the individuals' results were achieved. Thereby, the foundation was laid to implement an active iterative mechanism for group consensus seeking, which is capable of automatic unification of decision-makers' opinions. A mathematical optimization program was used with the purpose of reaching robust conclusions, and the consensus and agreement measures were defined in order to ensure convergence of the proposed procedure. Several robustness metrics were also defined to allow for identification of alternatives suitable for automatic/manual reassignment.

In addition, the influence of the noncompensatory veto and discordance thresholds on the criteria weights was discussed. An approach for automatic weight derivation was proposed. It computes the selective strengths of criteria and transforms them into a pairwise comparison matrix, which can be adjusted by the decision-maker to correctly reflect his personal beliefs.

The weight derivation approach has been recently evaluated according to a comprehensive experimental model. Simulation has been performed with regard to four independent variables - applied method, number of alternatives, probability of veto, and distribu- 
tion - as well as six dependent variables - sensitivity to values of input parameters, richness/extremness of weight discriminating information, proportion of rank perturbations in weak criteria orders between different applied methods, sensitivity to small changes of discordance indices, and sensitivity to adding either new alternatives or copies of existing alternatives. The results of the study have indicated the ability of selective strengths to provide good approximations of criteria weights. It has also been proven that the linear transformation of selective strengths induces near consistent pairwise comparison matrices, and that slightly inconsistent matrices generate more accurate and richer information on criteria weights than those which are obtained with the exponential function. Details on this empirical study will be presented in a separate paper.

The evaluation of the consensus seeking procedure is currently work in progress. A general framework has been defined (Bregar, 2005), which consists of twelve interrelated variables: guidance by the system; conflict resolution; convergence of opinions; cognitive load during analysis; initial cognitive load; level of imprecision, indetermination and uncertainty; ability of learning; ability of asynchronous interaction; time taken; thoroughness of problem domain analysis; robustness; and accuracy. Because of its high complexity, the framework requires combined application of two different research techniques - action research and statistical experiments in a controlled laboratory environment.

The introduced methodological solutions for consensus seeking, agent based negotiation and automatic weight derivation may be used either integratively in the context of a coherent interactive procedure, or in isolation. Due to substantial extensiveness, their applications will be the subject of a follow up paper. Although the localization principle leads to significant benefits, the weight derivation procedure and the group consensus seeking mechanism will be adapted to the other more general kinds of decision analyses as well. Particularly the case of ranking alternatives from the best to the worst ones will be considered within the scope of further research work.

\section{References}

Azar, S., J.-M. Azar, and J.-M. Hauglustaine (2001). Multicriteria and multiple actors tool aiding to optimise building envelope at the architectural sketch design. Informatica, 12(1), 3-24.

Bichler, M., G. Kersten, and S. Strecker (2003). Towards a structured design of electronic negotiation. Group Decision and Negotiation, 12(4), 311-335.

Bisdorff, R. (2000). Logical foundation of fuzzy preferential systems with application to the ELECTRE decision aid methods. Computers \& Operations Research, 27(7-8), 673-687.

Brans, J.P., C. Macharis, and B. Mareschal (1997). The GDSS PROMETHEE Procedure. STOOTW/277, V.U.B., Bruxelles.

Brans, J.P., and Ph. Vincke (1985). A preference ranking organisation method: The PROMETHEE method for multiple criteria decision-making. Management Science, 31(6), 647-656.

Bregar, A. (2005). Extension of the aggregation/disaggregation principle to computer-guided convergent group decision-making processes. In: Proceedings of Joint Workshop on Decision Support Systems, Experimental Economics and e-Participation. pp. 95-107.

Colson, G. (2000). The OR's prize winner and the software ARGOS: How a multijudge and multicriteria ranking GDSS helps a jury to attribute a scientific award. Computers \& Operations Research, 27(7-8), 741-755.

Demart, S., L. Dias, and V. Mousseau (2006). Supporting groups in sorting decisions: Methodology and use of a multi-criteria aggregation/disaggregation DSS. Decision Support Systems, doi: 10.1016/j.dss.2006.06.002. 
Dias, L., and J. Clímaco (2000). ELECTRE TRI for groups with imprecise information on parameter values. Group Decision and Negotiation, 9(5), 355-377.

Dias, L., and V. Mousseau (2003). IRIS: A DSS for multiple criteria sorting problems. Journal of Multi-Criteria Decision Analysis, 12(3), 285-298.

Dias, L., V. Mousseau, J. Figueira, and J. Clímaco (2002). An aggregation/disaggregation approach to obtain robust conclusions with ELECTRE TRI. European Journal of Operational Research, 138(2), 332-348.

Dzemyda, G., and T. Petkus (2001). Application of computer network to solve the complex applied multiple criteria optimization problems. Informatica, 12(1), 45-60.

Emond, E.J., and D.W. Mason (2002). A new rank correlation coefficient with application to the consensus ranking problem. Journal of Multi-Criteria Decision Analysis, 11(1), 17-28.

Espinasse, B., G. Picolet, and E. Chouraqui (1997). Negotiation support systems: A multi-criteria and multiagent approach. European Journal of Operational Research, 103(2), 389-409.

Eum, Y.S., K.S. Park, and S.H. Kim (2001). Establishing dominance and potential optimality in multi-criteria analysis with imprecise weight and value. Computers \& Operations Research, 28(5), 397-409.

Figueira, J., and B. Roy (2002). Determining the weights of criteria in the ELECTRE type methods with a revised Simos' procedure. European Journal of Operational Research, 139(2), 317-326.

Herrera, F., E. Herrera-Viedma, and F. Chiclana (2001). Multiperson decision-making based on multiplicative preference relations. European Journal of Operational Research, 129(2), 372-385.

Herrera-Viedma, E., F. Herrera, and F. Chiclana (2002). A consensus model for multiperson decision making with different preference structures. IEEE Transactions on Systems, Man and Cybernetics - Part A: Systems and Humans, 32(3), 394-402.

Huynh, V.-N., Y. Nakamori, T.-B. Ho, and T. Murai (2006). Multiple attribute decision making under uncertainty: The evidential reasoning approach revisited. IEEE Transactions on Systems, Man and Cybernetics Part A: Systems and Humans, 36(4), 804-822.

Jabeur, K., J.-M. Martel, and S. Ben Khélifa (2004). A distance-based collective preorder integrating the relative importance of the group's members. Group Decision and Negotiation, 13(4), 327-349.

Jacquet-Lagrèze, E., and Y. Siskos (2001). Preference disaggregation: 20 years of MCDA experience. European Journal of Operational Research, 130(2), 233-245.

Jaszkiewicz, A., and A.B. Ferhat (1999). Solving multiple criteria choice problems by interactive trichotomy segmentation. European Journal of Operational Research, 113(2), 271-280.

Jaszkiewicz, A., and R. Slowinski (1997). Outranking-based interactive exploration of a set of multicriteria alternatives. Journal of Multi-Criteria Decision Analysis, 6(1), 93-106.

Keeney, R.L., and H. Raiffa (1976). Decisions with Multiple Objectives: Preferences and Value Tradeoffs. John Willey \& Sons, New York.

Kim, H. (2001). An XML-based modeling language for the open interchange of decision models. Decision Support Systems, 31(4), 429-441.

Larichev, O. (2001). Method ZAPROS for multicriteria alternatives ranking and the problem of incomparability. Informatica, 12(1), 89-100.

Leyva-López, J.C., and E. Fernández-González (2003). A new method for group decision support based on ELECTRE III methodology. European Journal of Operational Research, 148(1), 14-27.

Lomuscio, A.R., M. Wooldridge, and N.R. Jennings (2003). A classification scheme for negotiation in electronic commerce. Group Decision and Negotiation, 12(1), 31-56.

Macharis, C., J. Springael, K. De Brucker, and A. Verbeke (2003). PROMETHEE and AHP: The design of operational synergies in multicriteria analysis. Strengthening PROMETHEE with ideas of AHP. European Journal of Operational Research, 153(2), 307-317.

Mateos, A., A. Jiménez, and S. Ríos-Insua (2003). Modelling individual and global comparisons for multiattribute preferences. Journal of Multi-Criteria Decision Analysis, 12(2-3), 177-190.

Matsatsinis, N.F., E. Grigoroudis, and A.P. Samaras (2005). Aggregation and disaggregation of preferences for collective decision-making. Group Decision and Negotitation, 14(3), 217-232.

Matsatsinis, N.F., and A.P. Samaras (2001). MCDA and preference disaggregation in group decision support systems. European Journal of Operational Research, 130(2), 414-429.

Miettinen, K., and P. Salminen (1999). Decision-aid for discrete multiple criteria decision making problems with imprecise data. European Journal of Operational Research, 119(1), 50-60.

Moshkovich, H.M., A.I. Mechitov, and D.L. Olson (2002). Ordinal judgments in multi-attribute decision analysis. European Journal of Operational Research, 137(3), 625-641. 
Mousseau, V., and L. Dias (2003). Valued outranking relations in ELECTRE providing manageable disaggregation procedures. European Journal of Operational Research, 156(2), 467-482.

Mousseau, V., and R. Slowinski (1998). Inferring an ELECTRE TRI model from assignment examples. Journal of Global Optimization, 12(2), 157-174.

Mousseau, V., R. Slowinski, and P. Zielniewicz (2000). A user-oriented implementation of the ELECTRE TRI method integrating preference elicitation support. Computers \& Operations Research, 27(7-8), 757-777.

Ngo The, A., and V. Mousseau (2002). Using assignment examples to infer category limits for the ELECTRE TRI method. Journal of Multi-Criteria Decision Analysis, 11(1), 29-43.

Peldschus, F., and E.K. Zavadskas (2005). Fuzzy matrix games multi-criteria model for decision-making in engineering. Informatica, 16(1), 107-120.

Petrovsky, A. (2001). Method for approximation of diverse individual sorting rules. Informatica, 12(1), 109118.

Raiffa, H., J. Richardson, and D. Metcalfe (2002). Negotiation Analysis: The Science and Art of Collaborative Decision Making. Belknap Harvard University Press.

Rauschmayer, F. (2001). Philosophical aspects of incommensurability and incomparability. Informatica, 12(1), $119-132$.

Ribeiro, R.A., and R.A. Marques Pereira (2003). Generalized mixture operators using weighting functions: A comparative study with WA and OWA. European Journal of Operational Research, 145(2), 329-342.

Roberts, R., and P. Goodwin (2002). Weight approximations in multi-attribute decision models. Journal of Multi-Criteria Decision Analysis, 11(6), 291-303.

Rogers, M., and M. Bruen (1998). Choosing realistic values of indifference, preference and veto thresholds for use with environmental criteria within ELECTRE. European Journal of Operational Research, 107(3), $542-551$.

Rogers, M., and M. Bruen (1998). A new system for weighting environmental criteria for use within ELECTRE III. European Journal of Operational Research, 107(3), 552-563.

Roy, B. (1991). The outranking approach and the foundations of ELECTRE methods. Theory and Decision, 31(1), 49-73.

Roy, B. (1996). Multicriteria Methodology for Decision Aiding. Kluwer Academic Publishers, Dordrecht.

Saaty, T.L. (1980). The Analytic Hierarchy Process. McGraw-Hill, New York.

Salo, A.A., and R.P. Hämäläinen (2001). Preference ratios in multiattribute evaluation (PRIME) - Elicitation and decision procedures under incomplete information. IEEE Transactions on Systems, Man and Cybernetics - Part A: Systems and Humans, 31(6), 533-545.

Saltelli, A., S. Tarantola, and K. Chan (1999). A role for sensitivity analysis in presenting the results from MCDA studies to decision makers. Journal of Multi-Criteria Decision Analysis, 8(3), 139-145.

Saltenis, V. (1998). Global sensitivity analysis of infection spread, radar search and multiple criteria decision models. Informatica, $9(2), 235-252$.

Stewart, T.J., and F.B. Losa (2003). Towards reconciling outranking and value measurement practice. European Journal of Operational Research, 145(3), 645-659.

Thiel, T., and T. Mroz (2001). Application of multi-criterion decision aid method in designing heating systems for museum buildings. Informatica, 12(1), 133-146.

Triantaphyllou, E. (2000). Multi-Criteria Decision Making Methods: A Comparative Study. Kluwer Academic Publishers, Dordrecht.

Triantaphyllou, E., and K. Baig (2005). The impact of aggregating benefit and cost criteria in four MCDA methods. IEEE Transactions on Engineering Management, 52(2), 213-226.

Turoff, M., and S. Hiltz (1996). Computer Based Delphi Processes. http://eies.njit.edu/ turoff/.

Vincke, Ph. (1999). Robust solutions and methods in decision aid. Journal of Multi-Criteria Decision Analysis, 8(3), 181-187.

Von Winterfeldt, D., and W. Edwards (1986). Decision Analysis and Behavioral Research. Cambridge University Press, Cambridge.

W3C (2006). W3C Recommendation on the Extensible Markup Language (XML) 1.0, Fourth Edition. http://www.w3 .org/TR/2006/REC-xml-20060816/.

Zavadskas, E.K., A. Zakarevicius, and J. Antucheviciene (2006). Evaluation of ranking accuracy in multicriteria decisions. Informatica, 17(4), 601-618.

Zeleny, M. (1982). Multiple Criteria Decision Making. McGraw-Hill, New York. 
Zhang, G., and J. Lu (2003). An integrated group decision-making method dealing with fuzzy preferences for alternatives and individual judgments for selection criteria. Group Decision and Negotiation, 12(6), 501515.

Zimmermann, H.-J. (1996). Fuzzy Set Theory - and Its Applications. Kluwer Academic Publishers, Dordrecht. Zopounidis, C., and M. Doumpos (2002). Multicriteria classification and sorting methods: A literature review. European Journal of Operational Research, 138(2), 229-246.

A. Bregar is a PhD candidate and a teaching assistant at the University of Maribor, Faculty of Electrical Engineering and Computer Science. He received BSc and MSc degrees in computer and information science in 1999 and 2002, respectively. He is the author of over 20 scientific and professional papers. The areas of his research work include decision support systems, intelligent systems, multi-criteria decision analysis, negotiation analysis, operations research, computer supported cooperative work, information systems development and XML technologies.

J. Györkös, PhD in computer science and informatics (1992), currently works as a full professor at the University of Maribor, Faculty of Electrical Engineering and Computer Science with teaching subjects on implications of information society and media convergence. His other research interests and academic background span from requirements management in software engineering, risk management to decision support systems. From 2001 to 2004 he spent one full mandate - 4 years - with the Government of Republic of Slovenia as a state secretary (deputy minister) in information society; during that time Slovenia joined the EU and he was responsible for the policy, ICT development and legislative initiatives on the information society area.

M.B. Jurič holds a $\mathrm{PhD}$ in computer and information science. He is an associate professor at the University of Maribor. He has authored several books, including Business Process Execution Language for Web Services, Best Practices for SOA-Based Integration and Composite Applications Development, J2EE EAI, Professional EJB, J2EE Design Patterns Applied, and .NET Serialization Handbook. He has published chapters in More Java Gems (Cambridge University Press) and in Technology Supporting Business Solutions (Nova Science Publishers). Matjaz has been involved in several large-scale IT projects. In cooperation with IBM Java Technology Centre, he worked on performance analysis and optimization of RMI-IIOP, integral part of the Java platform. He is a member of BPEL Advisory Board. 


\section{Interaktyvi kaupimo/padalinimo rikiavimo procedūra grupinei sprendimo analizei, pagrista slenksčio modeliu}

Andrej BREGAR, József GYÖRKÖS, Matjaž B. JURIČ

Šiame straipsnyje pristatyta nauja daugelio kriterijų sprendimu prièmimo procedūra, kuri surenka pirmenybės informaciją slenksčio modelyje. Ji yra pagrịsta rikiavimo analize, apribota lokalizavimo principo, leidžiančio sprendimo modelio prisitaikymą ir sumažinančio sprendiku pažinimo perkrovima. Tai igalina tris koncepcijas, kurios anksčiau buvo nepakankamai palaikomos kitu metodụ: pusiau automatinis kriteriju svoriu išvedimas priklausomai nuo atrankos pasekmiu bei veto slenksčiu, konverguojanti grupinio sutarimo paieška ir autonominės daugelio agentu derybos. Tarpusavyje priklausantys principai yra patikrinti ir realizacijos sprendimai pateikti. 